\title{
Miocarditis aguda como simuladora de infarto con ST elevado
}

\section{Acute myocarditis as simulator of an acute myocardial infarction}

\author{
Gabriela Santamaría-Naranjo* y Nelson E. Amores-Arellano
}

Servicio de Cardiología, Hospital Carlos Andrade Marín. Quito, Ecuador

La miocarditis es una enfermedad inflamatoria del miocardio y una de las principales causas de miocardiopatía dilatada, insuficiencia cardíaca y muerte súbita. Se calcula una incidencia de 22/100,000 personas, es decir, 1.5 millones de casos en la población mundial $\left(2013^{1}\right)$ y representa 5 a $22 \%$ de las muertes súbitas cardíacas en individuos jóvenes².

Para su definición y diagnóstico existen criterios clínicos, histopatológicos e inmunológicos. Los hallazgos histopatológicos que describió Dallas la definen como la presencia de infiltrados inflamatorios en el miocardio, en relación con una degeneración miocitaria y necrosis de causa no isquémica. Además, se han formulado algunos criterios inmunohistoquímicos: presencia en el miocardio de al menos 14 leucocitos $/ \mathrm{mm}^{2}$, entre ellos hasta 4 monocitos $/ \mathrm{mm}^{2}$ y detección de 7 o más linfocitos T CD3 positivos ${ }^{3}$.

Las causas más frecuentes son las infecciones virales (en particular enterovirus), bacterianas, micóticas y parasitarias. Se han descrito también causas no infecciosas, entre ellas enfermedades autoinmunitarias, hipersensibilidad a fármacos y toxinas ${ }^{3}$.

Su presentación clínica es variable, desde pacientes apenas sintomáticos hasta cuadros clínicos que semejan síndromes coronarios agudos o trastornos eléctricos, y se han descrito incluso casos que desarrollan insuficiencia cardíaca aguda y choque cardiogénico; esto dificulta el diagnóstico temprano, por lo que son de vital importancia para el diagnóstico los estudios electrocardiográficos y de imagen.
Se presenta el caso de un paciente masculino de 39 años con antecedentes de consumo de cocaína y marihuana, quien consulta por angina en reposo. Se lo valoró de forma inicial en un centro de segundo nivel, con 6 horas de evolución; el electrocardiograma (Fig. 1) mostró supradesnivel del ST en la cara anterior, por lo que se estableció un infarto con ST elevado; se instituyó tratamiento fibrinolítico sin lograr cambios en el trazado ni alivio del dolor. Se lo transfirió de inmediato al centro de los autores para practicar una intervención coronaria percutánea de rescate. La coronariografía mostró arterias coronarias sanas con flujo TIMI III.

La evaluación ecocardiográfica inicial evidenció hipocinesia lateral e inferolateral, fracción de expulsión del ventrículo izquierdo de $58 \%$ y disfunción diastólica GII. La curva de troponina mostró un comportamiento creciente hasta valores de $300 \mathrm{~N}$.

Con esta evidencia se realizó una resonancia magnética cardíaca contrastada (Fig. 2) que mostró zonas de edema y realce tardío intramiocárdico en las caras inferior y laterales consistentes con miocarditis. Con base en este diagnóstico se inició tratamiento vasodilatador con inhibidor ECA y betabloqueador con respuesta clínica favorable.

Se estableció seguimiento del paciente durante un año y en la actualidad se halla asintomático y con evaluación ecocardiográfica que muestra un ventrículo izquierdo sin trastornos de motilidad, fracción de expulsión de 64\% y función diastólica normal.
Correspondencia:

*Gabriela Santamaría-Naranjo

E-mail: gabricsantamaria@gmail.com
Disponible en internet: 10-02-2020 Arch Cardiol Mex. 2020;90(3):351-353 www.archivoscardiologia.com 1405-9940/@ 2020 Instituto Nacional de Cardiología Ignacio Chávez. Publicado por Permanyer. Este es un artículo open access bajo la licencia CC BY-NC-ND (http://creativecommons.org/licenses/by-nc-nd/4.0/). 


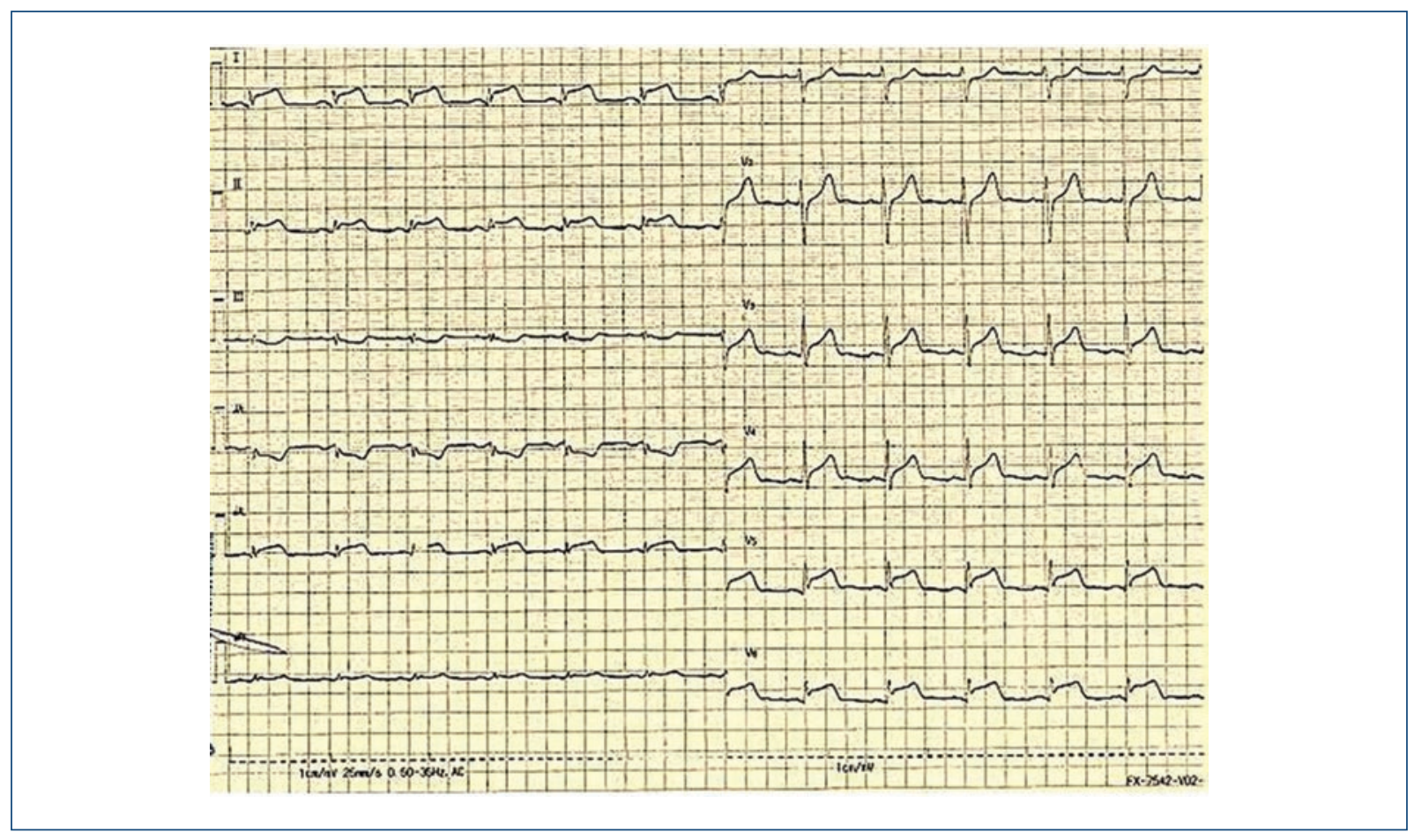

Figura 1. Electrocardiograma de 12 derivaciones.

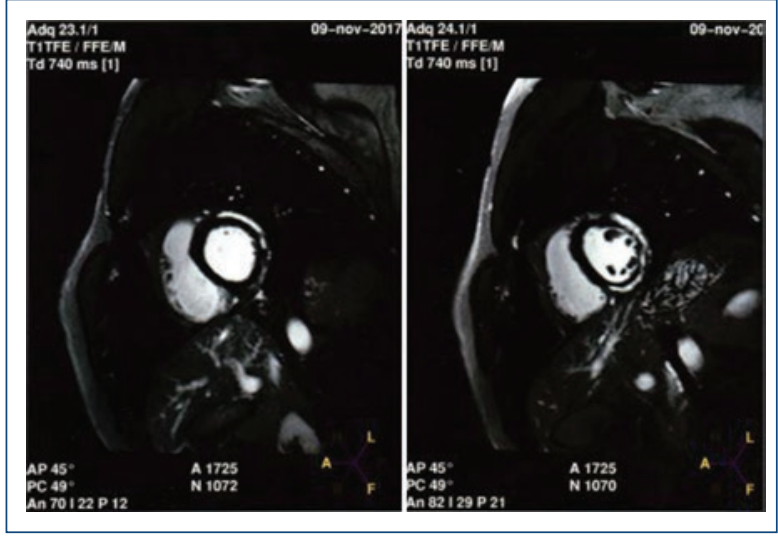

Figura 2. Resonancia magnética cardíaca con realce tardío con gadolinio.

La miocarditis puede simular un infarto agudo de miocardio por su presentación clínica, hallazgos electrocardiográficos y movilización de marcadores de citólisis miocárdica. En un estudio de 45 pacientes con sospecha de síndrome coronario agudo y una angiografía coronaria normal, 35 (78\%) tuvieron miocarditis difusa o focal confirmada por estudio de imagen 4 .

Los hallazgos ecocardiográficos incluyen trastornos difusos o regionales de motilidad, disfunción sistólica y cambios en la geometría del ventrículo izquierdo también frecuentes en la isquemia aguda.

La resonancia magnética cardíaca se ha convertido en el método de imagen de elección para el diagnóstico de la miocarditis, dado que ofrece información morfológica, funcional y de caracterización tisular de manera no invasiva. Los hallazgos adquieren mayor sensibilidad a partir del séptimo día de evolución de la enfermedad.

La resonancia magnética cardiovascular puede detectar edema como un aumento de intensidad en T2, un hallazgo que puede ser focal o difuso. La hiperemia se muestra como un aumento de la captación temprana de gadolinio en T1; y la necrosis y fibrosis miocárdica crean un patrón de realce tardío en parches sin respetar territorio vascular, lo que altera el subepicardio sin modificar el subendocardio. Estas características permiten realizar la diferenciación con afectación isquémica. La confirmación diagnóstica por resonancia magnética cardiovascular se realiza a través de los criterios de Lake y Louise y es positiva cuando hay al menos dos de los siguientes:

1. Aumento de la señal focal o difusa en las secuencias potenciadas en T2.

2. Realce temprano con gadolinio tras determinar el realce total absoluto o relativo del miocardio. 
3. Al menos un foco de realce tardío focal no isquémico ${ }^{5}$.

La presencia de dos de los tres criterios ofrece una precisión diagnóstica del $78 \%{ }^{5}$. El control de la miocarditis se basa en la administración de tratamiento vasodilatador en función del grado de disfunción sistólica del ventrículo izquierdo. Los individuos con choque cardiogénico, bloqueo $\mathrm{AV}$ de alto grado, arritmias ventriculares sostenidas y falla del tratamiento tras las primeras dos semanas requieren biopsia endomiocárdica para establecer la positividad para marcadores virales y determinar de forma específica el tratamiento ${ }^{6}$.

Todo paciente con diagnóstico de miocarditis debe tener restricción para la actividad física por seis meses y la reintegración al ejercicio se realiza sólo después de evaluación ecocardiográfica que confirme la función ventricular izquierda normal.

La resonancia magnética cardíaca (RMC) es quizás el mejor método de imagen para controlar la evolución del daño miocárdico, pero no existían datos claros de su significado pronóstico en el seguimiento de los pacientes con diagnóstico de miocarditis hasta el estudio ITAMY (ITAlian study in MYocarditis) ${ }^{7}$, que incluyó a un total de 187 pacientes con sospecha de miocarditis y función sistólica preservada, sometidos a una RMC durante la fase aguda y luego a un control a los seis meses, con el cual se logró establecer de forma más precisa su evolución y riesgos.

En el seguimiento se constató que los pacientes en quienes el realce tardío aumentaba en la segunda RMC desarrollaban de forma significativa más episodios cardíacos (muerte de causa cardíaca, paro cardíaco recuperado, trasplante, asistencia ventricular, descarga del desfibrilador automático implantable apropiada y hospitalización por insuficiencia cardíaca); de igual forma, los pacientes que tenían un realce tardío sin edema mostraban peor desenlace que aquellos en los que persistía. Todo lo anterior lleva a recomendar la RMC a los seis meses como un estudio para el seguimiento en estos casos.

Por último, el pronóstico de los pacientes con miocarditis aguda varía en relación con el escenario clínico y el grado de disfunción ventricular izquierda, y puede evolucionar a múltiples entidades, como miocarditis crónica, miopericarditis, así como una grave complicación, la miocardiopatía inflamatoria normal o dilatada que se caracteriza por un ventrículo izquierdo dilatado con disfunción sistólica.

\section{Financiamiento}

Esta investigación no ha recibido ninguna beca específica de agencias de los sectores público, comercial, o sin ánimo de lucro.

\section{Conflicto de intereses}

Ninguno.

\section{Responsabilidades éticas}

Protección de personas y animales. Los autores declaran que para esta investigación no se han realizado experimentos en seres humanos ni en animales.

Confidencialidad de los datos. Los autores declaran que han seguido los protocolos de su centro de trabajo sobre la publicación de datos de pacientes.

Derecho a la privacidad y consentimiento informado. Los autores han obtenido el consentimiento informado de los pacientes o sujetos referidos en el artículo.

\section{Bibliografía}

1. Global Burden of Disease Study 2013 Collaborators T, Barber RM, Bell B Bertozzi-Villa A, Biryukov S, Bolliger I, et al. Global, regional, and national incidence, prevalence, and years lived with disability for 301 acute and chronic diseases and injuries in 188 countries, 1990-2013: a systematic analysis for the Global Burden of Disease Study 2013. Lancet (London, England) [Internet]. 2015 Aug 22 [cited 2018 Sep 12];386(9995):743-800. Available from: http://www.ncbi.nlm.nih.gov/pubmed/26063472

2. Shah N, Phelan DMJ. Myocarditis in the athlete - American College of Cardiology [Internet]. 2018 [cited 2018 Sep 12]. Available from: https:// www.acc.org/latest-in-cardiology/articles/2018/01/18/15/00/myocarditis-in-the-athlete

3. Domínguez F, Kühl U, Pieske B, García-Pavía P, Tschöpe C. Actualización sobre miocarditis y miocardiopatía inflamatoria: el resurgir de la biopsia endomiocárdica. Rev Esp Cardiol. 2016;69(2):178-187; Available from: http://dx.doi.org/10.1016/j.recesp.2015.10.018 0300-8932/2015 Sociedad Española de Cardiología. Publicado por Elsevier España, S.L.U. Todos los derechos reservados

4. Cooper LT. Clinical manifestations and diagnosis of myocarditis in adults - UpToDate. 2018 [cited 2018 Sep 12]; Available from: https://www. uptodate.com/contents/clinical-manifestations-and-diagnosis-of-myocarditis-in-adults/print

5. De Castro ABG, Martínez BC, Domínguez JF, Villafañe CG, Fernández-Golfín C. Miocarditis: diagnóstico y seguimiento con resonancia magnética. Radiología. 2013;55(4):294-304.

6. Yic C, Pontet J, Cancela M. Miocarditis fulminante, caso clínico. Rev Méd Urug 2015;31(1):53-57.

7. Donato G. Prognostic value of repeating cardiac magnetic resonance in patients with acute myocarditis, J Am Coll Cardiol. 2019;74(20):24392448. 